\title{
Cucurbit Extracts Augment Biofilm Formation by Probiotic Lactobacilli: An In Vitro Study
}

\author{
Irfan A, Asad Khan M, Syed Hassan M, Zafaryab M, Ahmad P and Moshahid A Rizvi M
}

Department of Biosciences, Genome Biology Lab, Jamia Millia Islamia Public University, India

\begin{abstract}
Gastrointestinal tract predominantly harbor trillions of microorganisms including probiotics which maintain enteric microbial homeostasis. Some dietary cucurbits were investigated for their effects on biofilm formation by Lactobacillus rhamnosus (L. rhamnosus), Lactobacillus plantarum (L. plantarum), Lactobacillus acidophilus (L. acidophilus), Escherichia coli (E. coli) and Salmonella enterica typhimurium (S. enterica typhi). Aqueous and methanol extracts of Lagenaria siceraria $(L s)$, Luffa cylindrica $(L c)$ and Cucurbita maxima $(C m)$ were prepared and evaluated their effective concentrations on these bacterial strains. Effective concentrations for methanol and aqueous extracts were found $93.60 \mu \mathrm{g} / \mathrm{mL}-115.40 \mu \mathrm{g} / \mathrm{mL}$ and $103.67 \mu \mathrm{g} / \mathrm{mL}-121.00 \mu \mathrm{g} / \mathrm{mL}$, respectively. For the both types of extracts toxicity was determined up to $1 \mathrm{mg} / \mathrm{mL}$ concentration and found no microbicidal effects on probiotic strains. However, marginal inhibition on the growth of biofilms of pathogenic bacteria was observed. Extracts were found to support growth of biofilms of probiotics. Ls and $L c$ exhibited marginal inhibition on biofilm formation by $E$. coli and $S$. enterica typhi. Therefore, on the basis of our results it may be said that Lagenaria siceraria (bottle guard) and Luffa cylindrica (sponge guard) are safe, non-toxic and may be recommended as nutraceutical.
\end{abstract}

Keywords: Biofilm; Probiotics; Cucurbits; Gut; Pathogens

\section{Introduction}

Nutraceuticals have gained a great insight in recent years due to their therapeutic implications. Secondary metabolites such as flavonoids, polyphenols and terpenoids are widespread in nature and are consumed as part of human diet. Polysaccharides of prebiotic nature are also used as therapeutic and prophylactic agents. These nutraceuticals have great potential to reduce antigenic and oxidative pressure in the gut system. The population of probiotics in the gut may be maintained and balanced by dietary intervention of adequate amounts of vegetables and fruits containing prebiotics [1]. Diet influences gut functions through alteration in microbial composition. Plant polysaccharides present in our diet are utilized by enteric bacteria, and contribute host-microbe mutualism [2]. Probiotics have been reported to be evolved with genetic and biochemical potential to encode enzymes for breaking down of complex dietary polysaccharides [3-6]. Therefore, supplementation of nutraceuticals with diet might be potential therapeutic strategies to inhibit the process of pathogenesis through probiotics. Metabolites of probiotics such as short chain fatty acids (SCFAs) have been reported to act as epigenetic drug to cure cancer $[7,8]$. Beside the presence of various secondary metabolites of therapeutic importance, dietary plants are rich source of prebiotics which synergistically modulate the enteric microbial ecosystem in conjunction with host derived factors. Dietary plants act directly on the host and indirectly through host associated microbial processing. The present study is in continuation of our previous studies in which various activities like antioxidant, cytoprotective and modulation of proton pumping ATPase activities have been targeted in presence of cucurbit extracts [9-12]. Here we intended to explore the effect of extracts on growth and biofilm formation of probiotics as well as pathogens in an in vitro microbial culture system.

\section{Materials and Methods}

\section{Chemicals, reagents, glassware and plastic wares}

Growth medium for maintenance of microbial culture listed in Table 1, were procured from HiMedia (Mumbai, India). Other chemical constituents used in microbial culture were purchased from HiMedia (Mumbai, India) and Merck (Mumbai, India). Microtiter plates, petri plates and all other plastic wares were also purchased from HiMedia. Some chemicals and reagents were procured from Sigma (Germany). Inulin was procured from SRL (India).

\section{Preparation of extracts}

Fresh fruit of cucurbits, $L s, L c$ and $C m$ were purchased from local market of Jamia Nagar, New Delhi and identified. The herbarium numbers of the identified fruits were marked as Ls-GBL402, Lc-GBL402, Cm-GBL402 and preserved in the record of Genome Biology Lab., Department of Biosciences, JMI, New Delhi, India. Aqueous extracts were prepared as previously described [9-12]. Methanol extracts were prepared by Soxhlet extraction method. Briefly, fresh and cleaned $L s, L c$ and $\mathrm{Cm}$ were homogenized, dried at $62^{\circ} \mathrm{C}$ in a sterile incubator, packed in Soxhlet apparatus and extracted with methanol for $72 \mathrm{~h}$. Extracts were kept in vacuum desiccators for further studies.

\section{Procurement and maintenance of microbial strains}

Some standard strains of probiotics and pathogenic bacteria (Table 1) were procured from Microbial Type Culture Collection (MTCC), Institute of Microbial Technology, Chandigarh, India. Econorm of Dr. Reddy's Laboratory (Hyderabad, India) containing lyophilized Saccharomyces boulardii (S. boulardii) was purchased from local medical store, Jamia Nagar, New Delhi. Organisms were cultured and maintained in suitable growth medium as described in the Table 1. Luria Bertani (LB) and YEPD (Yeast Extract, Peptone and Dextrose) were used for maintenance of organisms $[12,13]$.

${ }^{*}$ Corresponding author: Moshahid Alam Rizvi M, Department of Biosciences, Genome Biology Lab, Jamia Millia Islamia Public University, India, Tel: 919911661657; E-mail: rizvi_ma@yahoo.com

Received March 14, 2017; Accepted June 03, 2017; Published June 10, 2017

Citation: Irfan A, Khan MA, Hassan MS, Zafaryab M, Ahmad P, et al. (2017) Cucurbit Extracts Augment Biofilm Formation by Probiotic Lactobacilli: An In Vitro Study. J Microb Biochem Technol 9:122-125. doi: 10.4172/1948-5948.1000354

Copyright: (C) 2017 Irfan A, et al. This is an open-access article distributed under the terms of the Creative Commons Attribution License, which permits unrestricted use, distribution, and reproduction in any medium, provided the original author and source are credited. 


\section{Listing of phytochemcals identified from extracts}

GC-MS based identification of compounds were done (for detail please refer our previous publication) [9]. Compounds identified from extracts were enlisted in tables which might be involved in supporting biofilm formation (Tables 2-4). However the complete lists of the identified compounds are the integral part of the $\mathrm{PhD}$ research work of I.A [14].

\section{Effect of extracts on bacterial biofilm formation}

Biofilms of different bacterial strains as mentioned in the Table1

\begin{tabular}{|l|c|c|c|c|}
\hline \multicolumn{1}{|c|}{ Strains } & $\begin{array}{c}\text { MTCC } \\
\text { Code }\end{array}$ & $\begin{array}{c}\text { Growth } \\
\text { medium }\end{array}$ & Temperature (0C) & pH \\
\hline L. rhamnosus & 1408 & MRS & $30-37$ & $6.5 \pm 0.02$ \\
\hline L. plantarum & 1407 & MRS & $30-37$ & $6.5 \pm 0.02$ \\
\hline L. acidophilus & 447 & MRS & $30-37$ & $6.5 \pm 0.02$ \\
\hline E. coli & 723 & LB & $30-37$ & $7.4 \pm 0.02$ \\
\hline S. enterictyphunarium & 98 & LB & $30-37$ & $7.4 \pm 0.02$ \\
\hline S. boulardii & YEPD & & $30-37$ & $7.2 \pm 0.02$ \\
\hline
\end{tabular}

Table 1: Strains of probiotics and pathogenic bacteria used in this study.

\begin{tabular}{|l|l|l|}
\hline Compounds & Retention time & \% Occurrence \\
\hline Furan methanol & 3.824 & 3.35 \\
\hline Glycol diacetate & 4.061 & 1.9 \\
\hline 4-Pyrimidinol & 4.531 & 2.66 \\
\hline a-Angelic lactone & 5.696 & 6.89 \\
\hline 2-Hydroxy-gamma-butyrolactone & 8.079 & 1.61 \\
\hline 2,5-Dimethyl-4-hydroxy-3(2H)-furanone & 10.313 & 5.11 \\
\hline 2,5-Anhydro-1,6-dideoxyhexo-3,4-diulose & 10.48 & 1.14 \\
\hline Cyclopropyl carbinol & 10.897 & 2.36 \\
\hline 4-Nitro-3-oxobutyric acid, methyl ester & 12.478 & 5.84 \\
\hline Acetic acid, anhydride & 14.277 & 1.59 \\
\hline $\begin{array}{l}\text { 2-Furancarboxaldehyde,5-(hydroxymethyl)- } \\
\text { Hexanoic acid }\end{array}$ & 15.172 & 16.4 \\
\hline 3,5-difluorophenyl ester & 17.204 & 1.24 \\
\hline Ortho-Methoxyacetophenone & 18.265 & 3.42 \\
\hline 2-Deoxy-D-glactose & 22.466 & 10.5 \\
\hline Palmitic acid & 32.009 & 1.79 \\
\hline Phthalic acid, dioctyl ester & 42.064 & 6.66 \\
\hline
\end{tabular}

Table 2: Compounds identified from extract of Lagenaria siceraria $(L s)$ fruit (Bottle guard).

\begin{tabular}{|l|l|l|}
\hline Compounds & Retention time & \% Occurrence \\
\hline 2-Furanmethanol & 3.846 & 3.47 \\
\hline 2,4-Dimethylimidazole & 4.577 & 13.48 \\
\hline N-Methyltetrazole & 5.341 & 2.85 \\
\hline Cyclohexanone & 5.633 & 2.56 \\
\hline 2,5-Dimethyl-4-hydroxy-3(2H)-furanone & 10.389 & 6.08 \\
\hline 4-Nitro-3-oxobutyric acid, methyl ester & 12.468 & 9.07 \\
\hline $\begin{array}{l}\text { 4-Amino-1,2,5-oxadiazole-3- } \\
\text { carbohydrazonamide }\end{array}$ & 13.752 & 2.14 \\
\hline Hydroxymethylfurfurole & 14.727 & 2.94 \\
\hline 2-Furancarboxaldehyde, 5-(hydroxymethyl)- & 15.214 & 13.01 \\
\hline Sorbitol & 26.621 & 1.28 \\
\hline Palmitic acid & 32.105 & 8.44 \\
\hline Linoleic acid & 35.260 & 2.51 \\
\hline Linolenic acid & 35.383 & 1.33 \\
\hline Phthalic acid, dioctyl ester & 42.062 & 5.19 \\
\hline Stigmasterol & 52.289 & 1.21 \\
\hline
\end{tabular}

Table 3: Compounds identified from extract of Luffa cylindrica $(L C)$ fruit (Sponge guard).

\begin{tabular}{|l|l|l|}
\hline \multicolumn{1}{|c|}{ Compounds } & $\begin{array}{l}\text { Retention } \\
\text { time }\end{array}$ & \% Occurrence \\
\hline 2-Furanmethanol & 3.876 & 5.42 \\
\hline Ethylene glycol, diacetate & 4.092 & 1.65 \\
\hline 2-Pyrimidinol & 4.56 & 6.76 \\
\hline Methyltetrazole & 5.359 & 1.76 \\
\hline 3-Amino-5-methylisoxazole & 5.696 & 3.49 \\
\hline Corylon & 8.748 & 1.08 \\
\hline 2,5-Hexanedione & 10.106 & 1.03 \\
\hline 2,5-Dimethyl-4-hydroxy-3(2H)-furanone & 10.53 & 7.52 \\
\hline 4-Nitro-3-oxobutyric acid, methyl ester & 12.757 & 3.61 \\
\hline Pyruvic acid, ethyl ester & 14.33 & 1.26 \\
\hline Hydroxymethylfurfurole & 15.551 & 28.3 \\
\hline N-Acetylcaprolactam & 22.567 & 1.04 \\
\hline Palmitic acid & 32.148 & 9.21 \\
\hline Linoleic acid, methyl ester & 35.323 & 4.13 \\
\hline Linoleic acid & 35.403 & 1.19 \\
\hline
\end{tabular}

Table 4: Compounds identified form extract of Cucurbita maxima ( $\mathrm{Cm}$ ) (Pumpkin)

were allowed to be formed in presence and absence of different extracts in 96-well microtiter plate by adjusting approximate cells density of $10^{6} /$ well. Plates were kept in static incubator at $37^{\circ} \mathrm{C}$. Biofilms formed were carefully maintained by changing growth medium after every $24 \mathrm{~h}$ and observed under microscope. OD at $600 \mathrm{~nm}$ of unwashed (with media) and washed (thrice with PBS) was read respectively in a microplate reader (Bio-Rad, iMark, USA). At the end photograph of biofilms were taken employing inverted microscope (Camera Bio-Wizard, USA).

\section{Results and Discussion}

\section{Composition of phytochemicals present in extracts}

Lagenaria siceraria (Ls), Luffa cylindrica (Lc) and Cucurbita maxima $(\mathrm{Cm})$ are most easily digestible vegetable. These groups of plants have gained a great insight due to their nutritional and medicinal potentials [9-12]. Phytochemical evaluations of different extracts prepared from fruits of these plants were carried out and a part of these have recently been published [9]. Tables $2-4$ contain phytochemicals present in extracts of $L s, L c$ and $C m$ respectively which were identified based on GC-MS analysis [9]. Our previous work suggests that cucurbits contain a balanced composition of certain compounds which modulate nutrient uptake mechanisms to promote cell viability by protecting against oxidants and intracellular $\mathrm{pH}[9,11]$. This is the first attempt towards protecting the biofilm of beneficial microorganisms through dietary intervention of cucurbit extracts in in vitro microbial culture system. However, individual compounds need to be evaluated on biofilm formation by human associated health promoting probiotics before going towards formulation of cucurbits derived herbal medicine (Tables 2-4).

\section{Effect of extracts on probiotics}

Aqueous and methanol extracts of cucurbits were evaluated on three strains of probiotic Lactobacilli (Table 1) and one strain of Saccharomyces boulardii. However, a part of the work showing effects on growth dynamics of these strains have been published [11] (Table 5). In this paper, methanol extracts were evaluated on growth dynamics and biofilms development of all probiotic Lactobacilli listed in Table 1.

\section{Determination of effective concentration}

Growth dynamics: Microbial growth dynamics in presence and absence of extracts were evaluated. Both aqueous [11] and methanol extracts of cucurbit fruits were found to enhance growth of probiotic 
Citation: Irfan A, Khan MA, Hassan MS, Zafaryab M, Ahmad P, et al. (2017) Cucurbit Extracts Augment Biofilm Formation by Probiotic Lactobacilli: An In Vitro Study. J Microb Biochem Technol 9:122-125. doi: 10.4172/1948-5948.1000354

strains. The effective concentrations (ECs) listed in the Table 5 were used in growth curve studies of different strains of probiotics. However, higher concentration of extracts $(1 \mathrm{mg} / \mathrm{mL})$ was also examined to find out the toxic effect of it but no toxicity was observed. The effect of different extracts on all strains of probiotics was evaluated by the growth curve studies. Significant and pronounced effects were observed on all strains with lag phage of 4-8h (L. rhamnosus and L. plantarum), 6-9 h (L. acidophillus), 4-6 h (S. boulardii) and active exponential phase 8, 9-18 h before attaining stationary phase (Figure 1). Concentration of the extracts used for growth curve studies were their respective EC values. Inulin $(100 \mu \mathrm{g} / \mathrm{ml})$ was used as positive control. Significantly enhanced growth pattern were observed for all the three extracts on L. rhamnosus ${ }^{\star} 1408$, L. plantarum ${ }^{\star} 1407$, L. acidophillus ${ }^{\star} 447$ and S. boulardii in comparison of normal controls. Results of the present study support our previous study in which extracts were evaluated for their cytoprotective and proton pumping ATPase activities on human gastrointestinal cells and probiotics $[9,11]$. Furthermore, extracts were found to exhibit marginal growth inhibition of pathogenic bacteria (PhD thesis, Figure 4.3.1 from I-L; Page. No. 133) [14]. Therefore, it can be said that our study explain the importance and utility of dietary cucurbits suggesting the rigorous additional research towards exploring the health promoting and anti-aging efficacies of certain phytochemicals present in these group of fruits (Figure 1).
Biofilm formation: Bacteria exhibit two life forms-planktonic (independent free living bacteria) and sessile aggregate. Bacterial biofilms are sessile aggregate of single or mixed population of different types of bacteria. Biofilms formed by pathogenic bacteria have killed millions of peoples worldwide [15] whereas biofilms of beneficial bacteria such as probiotics have been implicated as protective barrier and provide colonization resistance against pathogenic bacteria [16]. Gut-associated biofilm of probiotic bacterial communities modulate enteric barrier functions and immunity [17]. Furthermore, biofilms of Lactobacilli have been reported to produce anti-pathogenic and antiinflammatory factors that confer specific health benefits [18]. Biofilms of different species of probiotic bacteria formed on the gut mucosa needs to be protected and maintained. This can be achieved by dietary intervention of antioxidant rich fruits, vegetables and prebiotics. Furthermore probiotic Lactobacilli form a biofilm along the enteric mucosal surface and mitigate the activities of opportunistic bacterial pathogens and exogenous pathogens as well. Cucurbit fruit extracts have been reported to contain anti-oxidant compounds and secondary metabolites of therapeutic importance. Probiotic Lactobacilli have been reported to be involved in modulation of barrier function of the gut. In the present study extracts were found to support growth and biofilm formation of probiotic Lactobacilli. Methanol extracts were found to enhance growth of probiotics and effective concentration was determined $(93.60 \mu \mathrm{g} / \mathrm{mL}-115.40 \mu \mathrm{g} / \mathrm{mL})$. These extracts were found

\begin{tabular}{|c|c|c|c|}
\hline Extract & L. rhamnosus 1408 & L. plantarum 1407 & L. acidophilus 447 \\
\hline M-Ls & $93.60 \pm 5.29$ & $100.96 \pm 4.27$ & $103.14 \pm 5.71$ \\
\hline M-Lc & $95.01 \pm 5.23$ & $108.71 \pm 2.87$ & $99.44 \pm 3.77$ \\
\hline M-Cm & $115.34 \pm 5.12$ & $115.40 \pm 7.17$ & $104.63 \pm 8.72$ \\
\hline Inulin & $107.02 \pm 1.49$ & $111.96 \pm 3.69$ & $102.42 \pm 8.27$ \\
\hline
\end{tabular}

Aqueous extracts of $L s: 103.67 \pm 6.11 ; L c: 106.33 \pm 7.02$ and $C m: 113.00 \pm 6.00$

Table 5: Effective concentration of methanolic extracts enhancing growth of probiotics strains
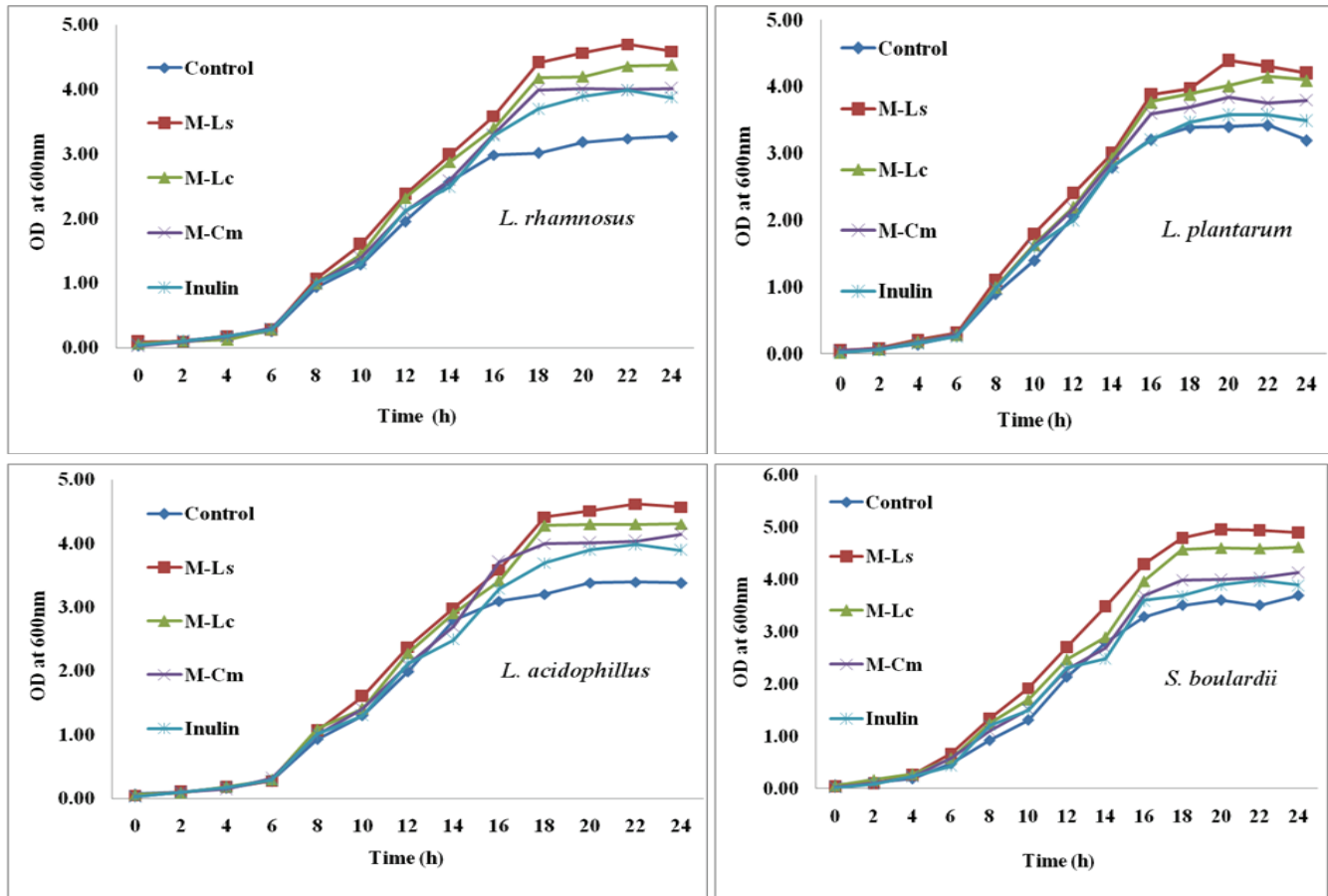

Figure 1: Strains of Lactobacilli and S. boulardii were grown on MRS and YEPD growth medium and optical density was read at the intervals of $2 \mathrm{~h}$ for $24 \mathrm{~h}$. 


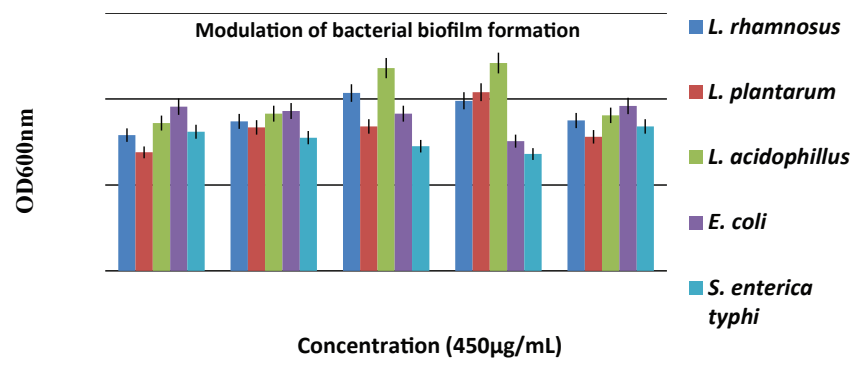

Figure 2: Effect of extracts on biofilm formation.

slightly more effective than aqueous extracts $(103 \mu \mathrm{g} / \mathrm{mL}-121 \mu \mathrm{g} /$ $\mathrm{mL}) .450 \mu \mathrm{g} / \mathrm{mL}$ of extracts was used for the study of biofilm formation because at this concentration maximum influx of nutrient was found to be utilized as evidenced in our previous study [11]. The methanol extracts of $L s$ and $L c$ were found more effective to show increased growth and biofilms of probiotics as compared to the normal control in which there were no supplement of extracts. The extract of $\mathrm{Cm}$ was also found less effective in comparison of $L s$ and $L c$ but more effective than positive control where inulin was used as prebiotic compound. $L s$ and $L c$ were found to exhibit inhibitory effect on developing biofilms of pathogenic E. coli and S. enterica typhi thereby suggesting prebiotic action and therapeutic implications of these extracts. Cm was found to support the formation of the biofilms of pathogenic bacteria (E. coli and $S$. enterica typhi) as compared to $L s, L c$ and inulin. Daily consumption of $\mathrm{Cm}$ vegetable fruit might be harmful and chronic infection of pathogenic enteric bacteria may be established. On the other hand Lagenaria siceraria $(L s)$ and Luffa cylindrica $(L c)$ were found safe due to not showing any support on growth of the biofilms formed by enteric pathogens (E. coli and S. enterica typhi). Individual compounds present in the extracts needs to be evaluated in animal model before formulation of therapeutic products. However, we therefore may suggest the consumption of $L s$ (bottle guard) and $L c$ (sponge guard) in adequate quantities would augment heath benefit through balancing and improving the function of enteric microbiome and host-microbes interactions (Figure 2).

\section{Conclusion}

Probiotic Lactobacilli form biofilm along the gut mucosal lining and modulate barrier function of the gut. Extracts of bottle and sponge guards were found to support biofilms of probiotic strains whereas inhibit pathogenic bacteria to form biofilms. Extract of Pumpkin was found to support growth and biofilms of pathogenic bacteria. Therefore, excluding pumpkin, two other cucurbits studied in the present research were found safe for health and can be consumed in adequate amount continuously. Cucurbit derived nutraceutical may be formulated for prophylactic and therapeutic uses to cure some ailments associated with the gut and systemic organs.

\section{Acknowledgement}

The authors report no conflict of interest. This study was financially supported by Defense Research and Development Organization (DRDO) and University Grant Commission (UGC), Government of India.

\section{References}

1. Roberfroid M, Gibson GR, Hoyles L, McCartney AL, Rastall R, et al. (2010) Prebiotic effects: Metabolic and health benefits. Br J Nutr 104: S1-S63.

2. Comstock LE (2009) Importance of glycans to the host-bacteroides mutualism in the mammalian intestine. Cell Host Microbe 18: 522-526.

3. Hooper LV, Wong MH, Thelin A, Hansson L, Falk PG, et al. (2001) Molecular aanalysis of commensal host-microbial relationships in the intestine. Science. 291:881-884.

4. Xu J, Bjursell MK, Himrod J, Deng S, Carmichael LK, et al. (2003) A genomic view of the human-Bacteroides thetaiotaomicron symbiosis. Science 299 2074-2076.

5. Pridmore RD, Berger B, Desiere F, Vilanova D, Barretto C, et al. (2004) The genome sequence of the probiotic intestinal bacterium Lactobacillus johnsoni NCC 533. Proc Natl Acad Sci 101: 2512-2525.

6. Tasse L, Bercovici J, Pizzut-Serin S, Robe P, Tap J, et al. (2010) Fundamenta metagenomics to mine the human gut microbiome for dietary fibercatabolic enzymes. Genome Res 20: 1605-1612.

7. Canani RB, Costanzo MD, Leone L (2012). The epigenetic effects of butyrate: Potential therapeutic implications for clinical practice. Clin Epigenet.

8. Marks PA, Richon VM, Miller T, Kelly WK (2004) Histone deacetylase inhibitors Adv Cancer Res. 91: 137-168.

9. Ahmad I, Irshad M, Zafaryab M, Asad Khan M, Syed HM, et al. (2017) Phytochemical composition of extracts prepared from dietary cucurbits and their cytoprotective efficacies against hydrogen peroxide induced oxidative stress in GI Cell-INT407. J Plant Biochem Physiol 5: 181.

10. Ahmad I, Nasiruddin M, Khan MA, Zafaryab M, Mehdi SH, et al. (2016) Nutraceuticals as chemopreventive and therapeutic agents in gut associated pathogenesis. Biochem Anal Biochem 5: 298.

11. Ahmad I, Zafaryab M, Al-Fatlawi AA, Kazim Z, Perwez A, et al. (2013) Modulation of growth and proton pumping ATPase activity of probiotic Lactobacilli by dietary cucurbits. JPP 4: 217-222.

12. Ahmad I, Irshad M, Rizvi MMA (2011) Nutritional and medicinal potential of Lagenaria siceraria. Int J Veg Sc 17: 157-170.

13. De Mann Rogosa S (1960) A Medium for the cultivation of Lactobacilli. J App Microbiol 23: 130-135.

14. Ahmad I (2015) Evaluation of certain cucurbits against gut microflora and thei therapeutic implications in gut associated pathogenesis.

15. Bjarnsholt T (2013) The role of bacterial biofilms in chronic infections. APMIS Suppl. 136:1-51.

16. Mujagic Z, de Vos P, Boekschoten MV, Govers C, Pieters HH, et al (2017) The effects of Lactobacillus plantarum on small intestinal barrier function and mucosalgene transcription; a randomized double-blind placebo controlled trial.

17. Macfarlane S, Dillon JF (2007) Microbial biofilms in the human gastrointestinal tract. J Appl Microbiol 102: 1187-1196.

18. Jones ES, Versalovic J (2009). Probiotic Lactobacillus reuteri biofilms produce antimicrobial and anti-inflammatory factors. BMC Microbiol. 